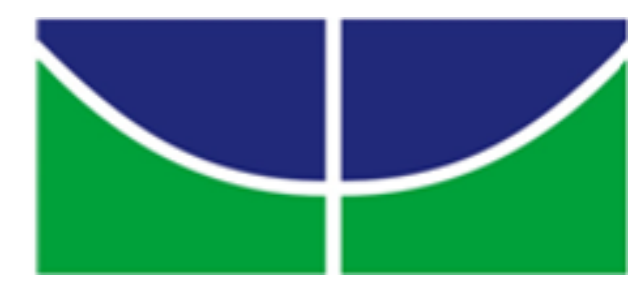

UNIVERSIDADE DE BRASÍLIA

Faculdade de Agronomia e Medicina Veterinária

Programa de Pós-Graduação em Saúde Animal

\title{
ALTERAÇÕES VENOGRÁFICAS PODAIS EM EQUINOS
}

JULIANA VIEIRA FLORES SALES

DISSERTAÇÃO DE MESTRADO EM SAÚDE ANIMAL

BRASÍLIA/DF

MARÇO/2017 


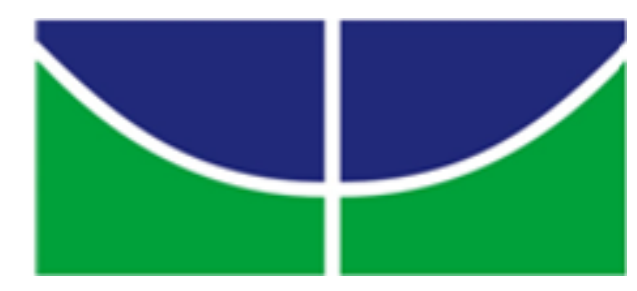

UNIVERSIDADE DE BRASÍLIA

Faculdade de Agronomia e Medicina Veterinária

Programa de Pós-Graduação em Saúde Animal

\title{
ALTERAÇÕES VENOGRÁFICAS PODAIS EM EQUINOS
}

\author{
JULIANA VIEIRA FLORES SALES
}

ORIENTADOR: ANTÔNIO RAPHAEL TEIXEIRA NETO

DISSERTAÇÃO DE MESTRADO EM SAÚDE ANIMAL

PUBLICAÇÃO: 140/2017

BRASÍLIA/DF

MARÇO/2017 


\title{
REFERÊNCIA BIBLIOGRÁFICA E CATALOGAÇÃO
}

Sales, J. V. F. Alterações venográficas podais em equinos. Brasília: Faculdade de Agronomia e Medicina Veterinária, Universidade de Brasília, 2017, 30 p. Dissertação de mestrado.

\begin{abstract}
Documento formal, autorizando reprodução desta dissertação de Mestrado para empréstimo ou comercialização, exclusivamente para fins acadêmicos; foi passado pelo autor à Universidade de Brasília e achase arquivado na secretaria do Programa. O autor reserva para si os outros direitos autorais, de publicação. Nenhuma parte desta dissertação de mestrado pode ser reproduzida sem a autorização por escrito do autor. Citações são estimuladas, desde que citada a fonte.
\end{abstract}

Sales, Juliana Vieira Flores

Alterações venográficas podais em equinos. / Juliana Vieira Flores Sales

Orientação de Antônio Raphael Teixeira Neto

Brasília, 2017. 30 p.:il.

Dissertação de mestrado (M) - Universidade de Brasília/ Faculdade de Agronomia e Medicina Veterinária, 2017.

1.Cavalo 2. Venograma 3. Vascularização do dígito 4. Claudicação I. Teixeira Neto, A. R. II. Doutor 


\title{
ALTRRACYOES VENOGRAFICAS PODAIS FM EQUTNOS
}

\author{
TULANA VIEIRA FLORES SALES
}

DASSERTACĀO DE MESTRADO SUBMETDA AO PROGRAMA DE POS GRADUACÃO EM SALDE ANIMAL. COMO PARTE DOS REQUISTTOS NECESSÁRIOS A OBTENCYÃO DO GRAU DE MESTRE EM SAUDE ANIMAL

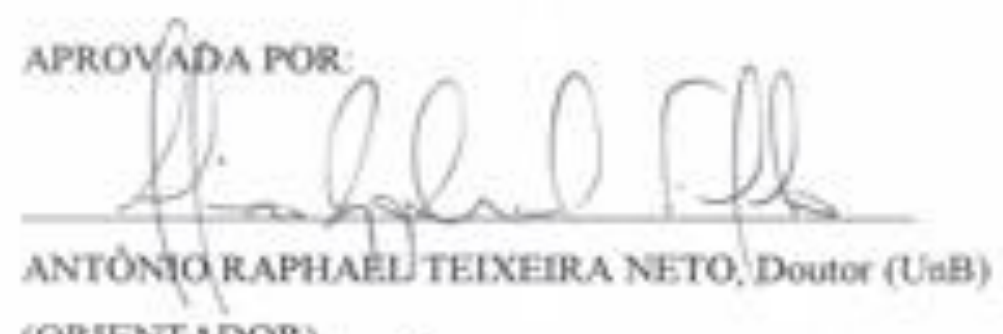

(ORIENTADOR)

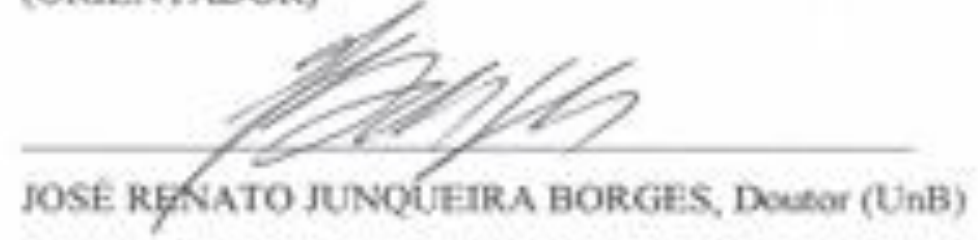

(EXAMINADOR INTERNO)

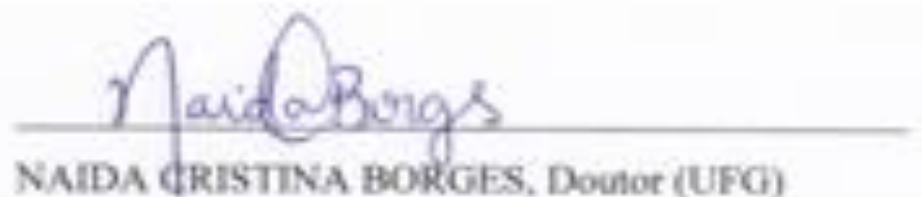

(EXAMINADOR EXTERNO)

RRAShLLMDE, 20 de Marşo de 2017 
"Mesmo quando tudo parece desabar, cabe a mim decidir entre rir ou chorar; ir ou ficar; desistir ou lutar; porque descobri, no caminho incerto da vida, que o mais importante é decidir"

(Cora Coralina) 


\section{AGRADECIMENTOS}

A Deus e a todos que olham por nós, gratidão por mais uma realização.

À minha família por todo apoio, conselhos e incentivo.

A aluna Alana e ao meu noivo Vitor, sem vocês eu jamais teria conseguido, obrigada pela ajuda impar em todas as etapas do projeto.

Ao professor Antônio Raphael Teixeira Neto, pelos ensinamentos, orientação e atenção nesta minha jornada, desde 2006, no Hospital Veterinário de Grandes Animais da Universidade de Brasília (Hvetão).

À grande equipe do Hvetão: professores, funcionários, médicos veterinários concursados, residentes e estagiários; pelo apoio, amizade, respeito e confiança.

A médica veterinária Ana Lourdes Arrais de Alencar Mota e ao professor Vitor Salvador Picão Gonçalves, por abrirem as portas do Laboratório de Epidemiologia Veterinária para me ajudar com toda a análise estatística do projeto.

Aos animais, seres sem igual. Obrigada por serem tão especiais na minha vida. 


\section{SUMÁRIO}

Página

LISTA DE ABREVIAÇÔES ....................................................................................... viii

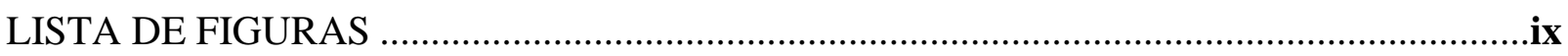

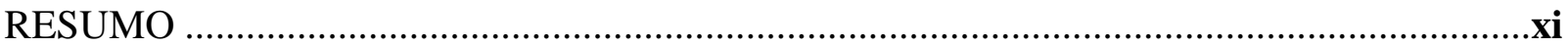

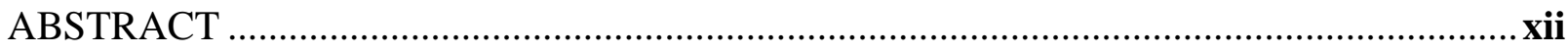

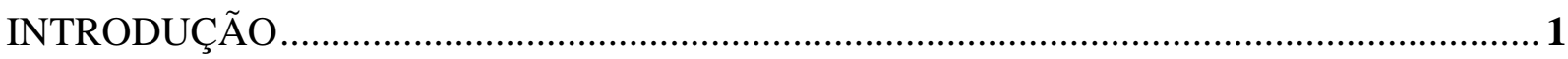

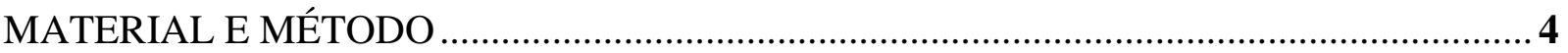

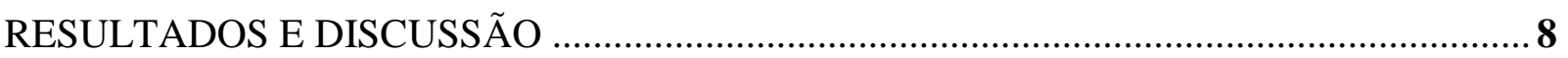

CONCLUSÃO

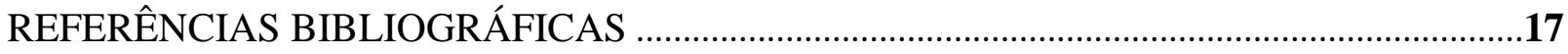




\section{LISTA DE ABREVIAÇÕES}

$\begin{array}{ll}\mathrm{Kg} & \text { Quilograma } \\ \mathrm{mg} & \text { Miligrama } \\ \mathrm{cm} & \text { Centímetros } \\ \% & \text { Por cento } \\ \mathrm{LM} & \text { Latero-medial } \\ \mathrm{mL} & \text { Mililitro } \\ \mathrm{Kv} & \text { Quilovot } \\ \mathrm{mA} & \text { miliAmpère } \\ \mathrm{n} & \text { Número de animais } \\ \mathrm{DP} & \text { Desvio padrão } \\ < & \text { Menor } \\ > & \text { Maior }\end{array}$




\section{LISTA DE FIGURAS}

Página

Figura 1 Artérias do dígito equino: (1) artéria digital palmar; (2) 2 ramo a falange proximal; (3) ramo ao coxim digital; (4) ramo dorsal a falange média; (5) ramo dorsal a falange distal; (6) artéria circunflexa. Modificado de PARKS (2003).

Figura 2 Membro torácico direito de equino submetido à venografia. 5 A: canulação da veia digital palmar lateral. B: Injeção de contraste à base de diatrozoato de meglumina.

Figura 3 Projeção LM de membro torácico direito de equino após 6 injeção de contraste à base de diatrozoato de meglumina.

Figura 4 Membros torácicos de equinos adultos não claudicantes (1) 8 e claudicantes (2,3) em projeção lateromedial. (1) Regiões de referência identificadas nos venogramas. (A) - arco terminal, (B) - vasos laminares dorsais, (C) - plexo coronário, (D) - vasos circunflexos e (E) - bulbo do talão. (2) - Venograma de membro torácico direito com diminuição de preenchimento em plexo coronário e vasos laminares dorsais. (3) - Venograma de membro torácico direito com diminuição de preenchimento em plexo coronário, vasos laminares dorsais, vasos circunflexos, arco terminal e bulbo do talão.

Figura 5 Representações gráficas (diagramas em caixas) das médias e desvios padrões do preenchimento por contraste radiográfico dos dígitos de equinos não claudicantes (Grupo 1) e claudicantes (Grupo 2). 
Figura 6 Venograma do membro torácico direito de animal com claudicação $3 / 5$ com pontuação total de 8 pontos (A), venograma do membro torácico esquerdo de animal com claudicação $4 / 5$ com pontuação total de 16 pontos (B). Setas apontam diminuição de perfusão, em (A): plexo coronário, vasos laminares dorsais, vasos circunflexos, arco terminal e bulbo do talão; em (B): vasos laminares dorsais, vasos circunflexos, arco terminal e bulbo do talão.

Figura 7 Fotos e venogramas dos membros torácicos direitos de dois 13 animais claudicantes. (A) animal com necrose de cartilagem alar. Setas apontam diminuição de perfusão em plexo coronário, vasos laminares dorsais, vasos circunflexos, arco terminal e bulbo do talão. (B) animal com laminite crônica. Setas apontam diminuição de perfusão em vasos laminares dorsais e vasos circunflexos.

Figura 8 Conformação do casco de três animais. (A) casco encastelado, (B) casco achinelado, (C) casco normal. 


\section{RESUMO}

Devido à característica e ao envolvimento vascular da pododermatite asséptica, a compreensão das ocorrências vasculares dentro do casco passou a ser o foco dos trabalhos científicos. A venografia começou a ser utilizada para este fim. É um método de diagnóstico por imagem minimamente invasivo, prático e essencial para acessar a circulação digital. Auxilia, portanto, médicos veterinários e ferradores na escolha da terapêutica apropriada para a restauração da circulação do digito; possuindo acurado valor prognóstico. No presente trabalho, para o estudo venográfico dos cascos de membros torácicos, foram utilizados 19 equinos com idade média de 9,5 \pm 4,4 anos, 12 machos e 07 fêmeas, sendo 11 animais com ausência de sinais clínicos de claudicação e 08 apresentando grau de claudicação de apoio, variando de 1 a 4/5, de acordo com método proposto pela AAEP. O objetivo do trabalho foi avaliar venogramas dos dígitos de animais claudicantes e não claudicantes, a partir da criação de escala semi-quantitativa de preenchimento por contraste radiográfico, nas seguintes regiões: arco terminal, vasos laminares dorsais a falange distal, plexo coronário, vasos circunflexos e bulbo do talão. Os dados obtidos foram submetidos à análise estatística ANOVA, complementados por TUKEY, com nível de significância $p<0,05$ para comparação entre todos os grupos (membro torácico esquerdo de animais não claudicantes, membro torácico direito de animais não claudicantes, membro torácico esquerdo de animais claudicantes e membro torácico direito de animais claudicantes). Obteve-se diferença estatística entre os venogramas dos animais claudicantes e não claudicantes, não importando o membro torácico utilizado para a análise. Isso significa dizer que o animal com deficiência de apoio apresenta menor perfusão do dígito. Quanto as regiões observadas, a diminuição de irrigação se fez presente com maior frequência no arco terminal, nos vasos circunflexos e nos vasos laminares dorsais.

TERMOS DE INDEXAÇÃO: Cavalo, venograma, vascularização do dígito, claudicação. 


\begin{abstract}
Due to the characteristic and vascular involvement of aseptic pododermatitis, the understanding of vascular occurrences within the foot became the focus of scientific work. The venography began to be used for this purpose. It is a minimally invasive, practical and essential diagnostic imaging method to access the digital circulation. It assists, therefore, veterinarians and farriers in the choice of the appropriate therapy for the restoration of the circulation of the digit, having an accurate prognostic value. In the present research, 19 horses with a mean age of $9.5 \pm 4.4$ years, 12 males and 07 females, were used for the venography study of the foot of the forelimbs, of which 11 without clinical signs of lameness and 08 presented a degree of claudication of support, varying from 1 to $4 / 5$, according to the method proposed by the AAEP.The purpose of this study was to evaluate the venograms of the digits of both, claudicating and non-claudicating animals, from the creation of a semi-quantitative scale filled by radiographic contrast data, of the following regions: terminal arch, dorsal laminar vessels to the distal phalanx, coronary plexus, circumflex vessels and heel perfusion. The gathered data were submitted to ANOVA statistical analysis, complemented by TUKEY, with significance level $\mathrm{p}<0.05$ for comparison between all groups (left thoracic limb of nonclaudicating animals, right thoracic limb of non-claudicating animals, left thoracic limb of claudicating animals and right thoracic limb of claudicating animals). There was statistical difference among the venograms of the claudicating and non-claudicating animals, regardless of the thoracic limb used for the analysis. This means that the animal with a support deficiency has lower perfusion of the digit. Regarding the regions observed, the decrease of irrigation was more frequently present in the terminal arch, circumflex vessels and dorsal laminar vessels.
\end{abstract}

INDEXING TERMS: Horse, venogram, vascularization of the digit, lameness. 


\section{INTRODUÇÃO}

O aparelho locomotor dos equinos é considerado de grande importância por constituir o sistema de sustentação e dinâmica da locomoção nesta espécie (THOMASSIAN, 2000). Nos equinos, os membros torácicos sustentam 60 a $65 \%$ de seu peso corporal, portanto, a maioria das lesões se concentram nestes membros. Delas, cerca de $95 \%$ se originam no carpo ou distal a ele (STASHAK, 2006).

A claudicação é conceituada como uma anormalidade no andar, ou seja, uma alteração funcional ou estrutural, de um ou mais membros ou do dorso, observada ao exame estático ou dinâmico. Sob circunstâncias normais a alteração na marcha do animal se aplica a lesões de origem dolorosa. Dentre os principais fatores estão os traumas, as anomalias congênitas ou adquiridas, a infecção, os distúrbios metabólicos, as alterações nervosas ou circulatórias (SPEIRS, 1999; BAXTER, 2011).

A claudicação de membro de apoio, ou seja, membro que sustenta o peso corporal, caracteriza-se por movimentos anormais da cabeça e do corpo à medida que o cavalo tenta redistribuir seu peso, assim, sempre que o casco do membro afetado toca o solo, recebendo a carga de peso, o animal levanta a cabeça, na tentativa de deslocar o centro de gravidade e diminuir o peso sobre o membro (SPEIRS, 1999).

Portanto, devido à falta de contato com o solo, o casco não desempenha algumas de suas funções como, por exemplo, absorção de impacto com o solo, suporte de peso corporal, resistência ao desgaste e auxilio no retorno sanguíneo da extremidade do membro locomotor (KANE et al, 1996; NICOLETTI et al., 2000).

Dentre as principais afecções que acometem os dígitos dos equinos podemos destacar a pododermatite asséptica difusa ou laminite. Esta enfermidade é considerada uma doença vascular periférica visto que durante seu desenvolvimento há diminuição da perfusão capilar no casco cursando com abertura de anastomoses arteriovenosas, isquemia, necrose de lâminas e dor (STASHAK, 2006). Devido à característica, à severidade e o envolvimento vascular da afecção em questão, o entendimento das alterações vasculares digitais passou a intigrar os pesquisadores e ser tema de seus estudos.

Em 1970, Coffman e colaboradores exporam a circulação do casco a partir da técnica de angiografia em equinos anestesiados, neste exame foi demonstrado a ocorrência de hipoperfusão laminar em processos de laminite. 
Redden (2001) desenvolveu a técnica de venografia com os animais mantidos em apoio quadrupedal. A injeção intravenosa de contraste foi possível pois a maioria das veias dos dígitos dos equinos não apresentam válvulas, produzindo assim uma imagem de circulação (POLLITT,2008).

Em relação à vascularização do dígito, sabe-se que os vasos sanguíneos são os responsáveis pelo fornecimento de nutrientes e pela remoção de resíduos, bem como, uma rota para substâncias químicas como hormônios e toxinas acessarem os tecidos do casco (DAVIES et al., 2007).

As artérias digitais palmares medial e lateral são responsáveis pelo suprimento sanguíneo arterial do dígito (BURG et al., 2007). Segundo Parks (2003), cada artéria digital palmar envia vários ramos (Figura 1).

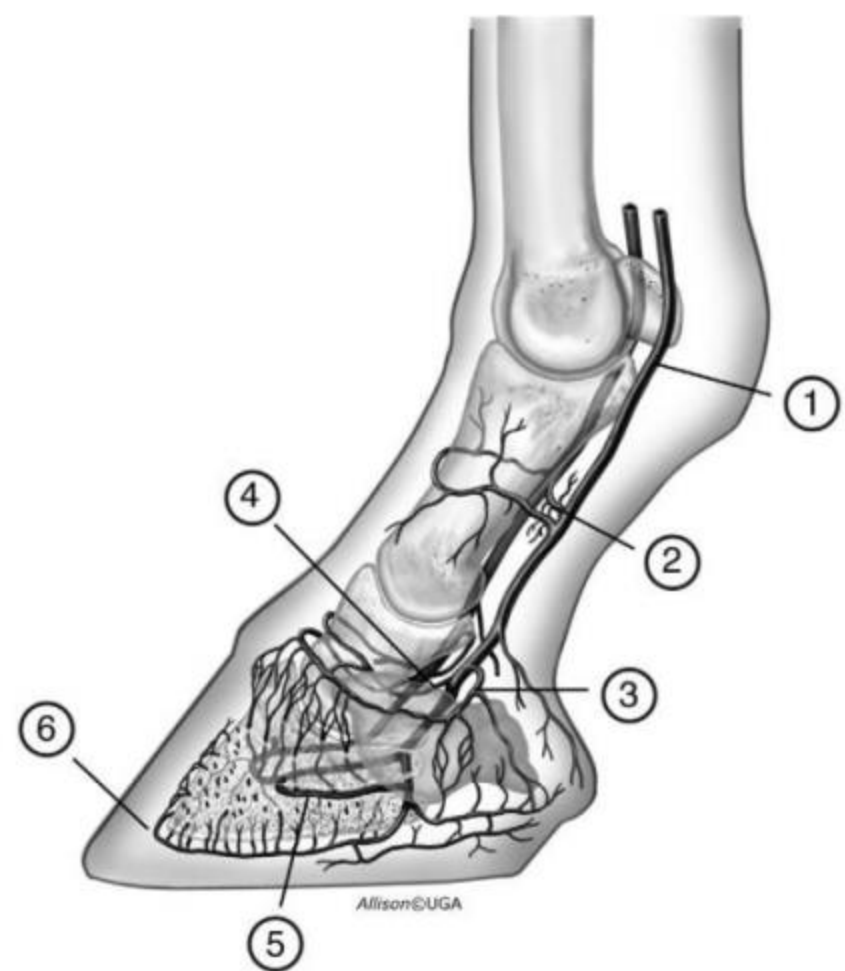

Figura 1: Artérias do dígito equino: (1) artéria digital palmar; (2) ramo a falange proximal; (3) ramo ao coxim digital; (4) ramo dorsal a falange média; (5) ramo dorsal a falange distal; (6) artéria circunflexa. Modificado de PARKS (2003). 
Além disso, as artérias digitais, para formar o arco terminal, atravessam, pelo forâmen solear, os canais soleares da falange distal e fazem uma anastomose com o vaso contralateral. Fato curioso consiste na irrigação da região laminar dorsal, de distal para proximal. Devido a esse fato, as artérias que estão localizadas nesta região são as últimas a serem per fundidas (STOKES et al., 2004) e, portanto, estão mais propensas a lesões por isquemia e reperfusão (PARKS, 2003).

Em relação a drenagem do digito, a diferença mais marcante em relação à irrigação é a presença de três plexos venosos interligados no casco: plexo coronário, plexo venoso dorsal na derme lamelar e o plexo venoso palmar no cório solear. Esses plexos são drenados pelas veias digitais medial e lateral (PARKS, 2003). Além disso, a circulação laminar conta com cerca de 50 a 500 anastomoses arteriovenosas por centímetro quadrado (ROBINSON, 1990).

A venografia é um método de diagnóstico por imagem minimamente invasivo, prático e capaz de revelar a saúde do sistema vascular. A venografia dos dígitos pode auxiliar, portanto, médicos veterinários e ferradores na escolha da terapêutica apropriada para a restauração da circulação do digito; possui acurado valor prognóstico; e monitora os pacientes, podendo ser repetido em 3 a 14 dias (REDDEN, 2001; RUCKER, 2010). As áreas de maior interesse na venografia são: o arco terminal, os vasos circunflexos, os vasos laminares dorsais, o plexo coronário e o bulbo do talão (REDDEN, 2001).

Diante disso, pretendeu-se no presente trabalho avaliar venogramas dos dígitos de animais claudicantes e não claudicantes, a partir da criação de escala semi-quantitativa de preenchimento por contraste radiográfico, em regiões pré-estabelecidas.

\section{MATERIAL E MÉTODO}

Foram utilizados 19 equinos com idade média de 9,5 4,4 anos, 12 machos e 07 fêmeas, com peso médio de $333,2 \pm 70,8 \mathrm{~kg}$, sendo onze animais com ausência de sinais clínicos de claudicação e oito animais apresentando claudicação. O grau de claudicação foi definido de acordo com o método de classificação proposto pela "American Association of Equine Practicioners (AAEP, 1996), para o estudo venográfico dos cascos dos membros torácicos. O trabalho foi avaliado e aprovado pelo Comitê de Ética no Uso Animal da Universidade de Brasília (CEUA-UnB) sob o protocolo 66740/2016. 
Para realização da venografia digital, cada equino teve o casco minuciosamente limpo, com água e sabão, a fim de se evitar qualquer artefato na imagem. Ademais, foi realizada tricotomia na região da quartela de ambos os membros torácicos para posterior punção da veia digital palmar lateral ou medial. A tricotomia foi realizada na face medial e lateral de cada quartela, pois se caso não houvesse sucesso na venopunção de uma face, a outra já estaria pronta.

O animal foi conduzido para uma superfície plana (tapete de borracha de $3 \mathrm{~cm}$ de espessura, previamente limpo). Após o posicionamento do animal em estação (apoio quadrupedal), fotografias dos cascos dos membros torácicos sobre a perspectiva de quatro ângulos (dorsal, medial, lateral e palmar) foram realizadas a fim de se evidenciar a conformação de cada casco.

Em seguida foi realizada a aferição da angulação dos cascos por meio de angulador específico e os sulcos centrais das ranilhas foram preenchidos com massa de modelar visando minimizar artefatos na imagem. O animal foi sedado com cloridrato de detomidina na dose de 0,01mglkg e um colaborador acostumado a lida com equinos, ficou responsável pela contenção do animal com cabresto, preocupando-se em manter a cabeça do mesmo sempre reta e alinhada para se evitar quaisquer preenchimentos anormais por apoio desigual nos membros após aplicação do contraste.

De acordo com a técnica descrita por Redden (2001) e por Rucker (2010), realizou-se anestesia perineural digital palmar lateral e ou medial na região da quartela $(3 \mathrm{~mL}$ de lidocaína $2 \%$ sem vasoconstritor, por ponto) e cada membro foi posicionado em bloco de madeira, para ser radiografado. Uma antissepsia cirúrgica foi realizada no local da venopunção com auxílio de iodopovidona degermante e álcool. Um torniquete foi fixado na base do boleto na altura dos ossos sesamóides proximais. Uma das veias digitais palmares foi canulada com auxílio de escalpe de calibre 21 e então injetou-se contraste a base de diatrozoato de meglumine $60 \%$ $\left(\right.$ Reliev $^{\circledR}$ ), dividido em duas seringas de $10 \mathrm{~mL}$, totalizando $20 \mathrm{~mL}$ de contraste (Figura 2). 


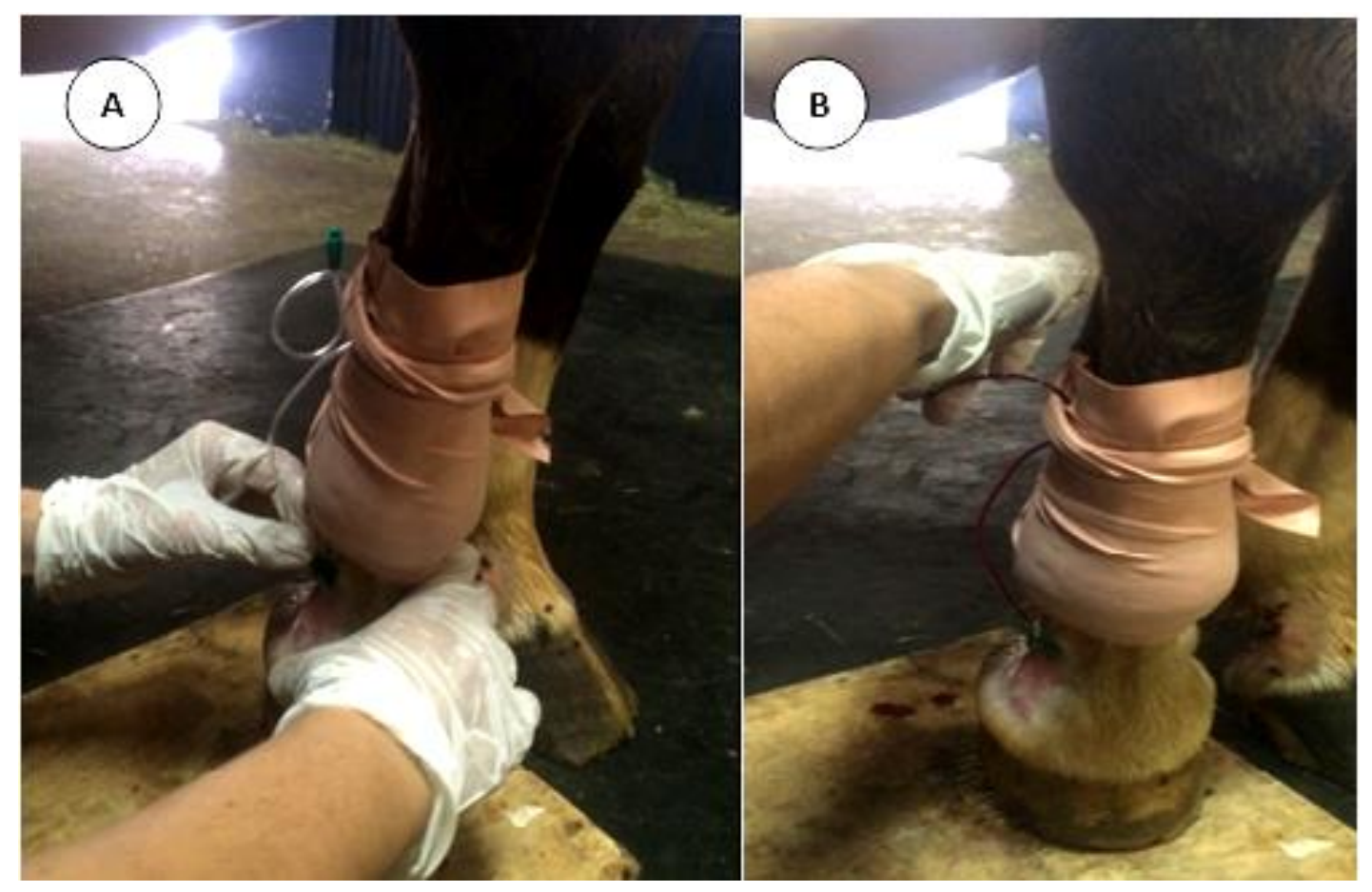

Figura 2: Membro torácico direito de equino submetido à venografia. A: canulação da veia digital palmar lateral. B: Injeção de contraste à base de diatrozoato de meglumina.

Logo após a aplicação do contraste, o membro era flexionado e novamente posicionado no bloco de madeira para realização de projeção lateromedial (LM) com auxílio de aparelho de radiologia (Poskom PXM-40BT) acoplado a um sistema de captação e digitalização de imagem CR 30X AGFA. Todo o procedimento, após a injeção do contraste durava em torno de de 45 segundos (Figura 3). Após radiografia, o cateter era retirado da veia e uma bandagem compressiva, realizada com iodopovidona tópica, gaze, algodão e atadura. O curativo era retirado no dia posterior e cada animal era monitorado por 96 horas, visando possível intervenção clínica no caso de flebite. 


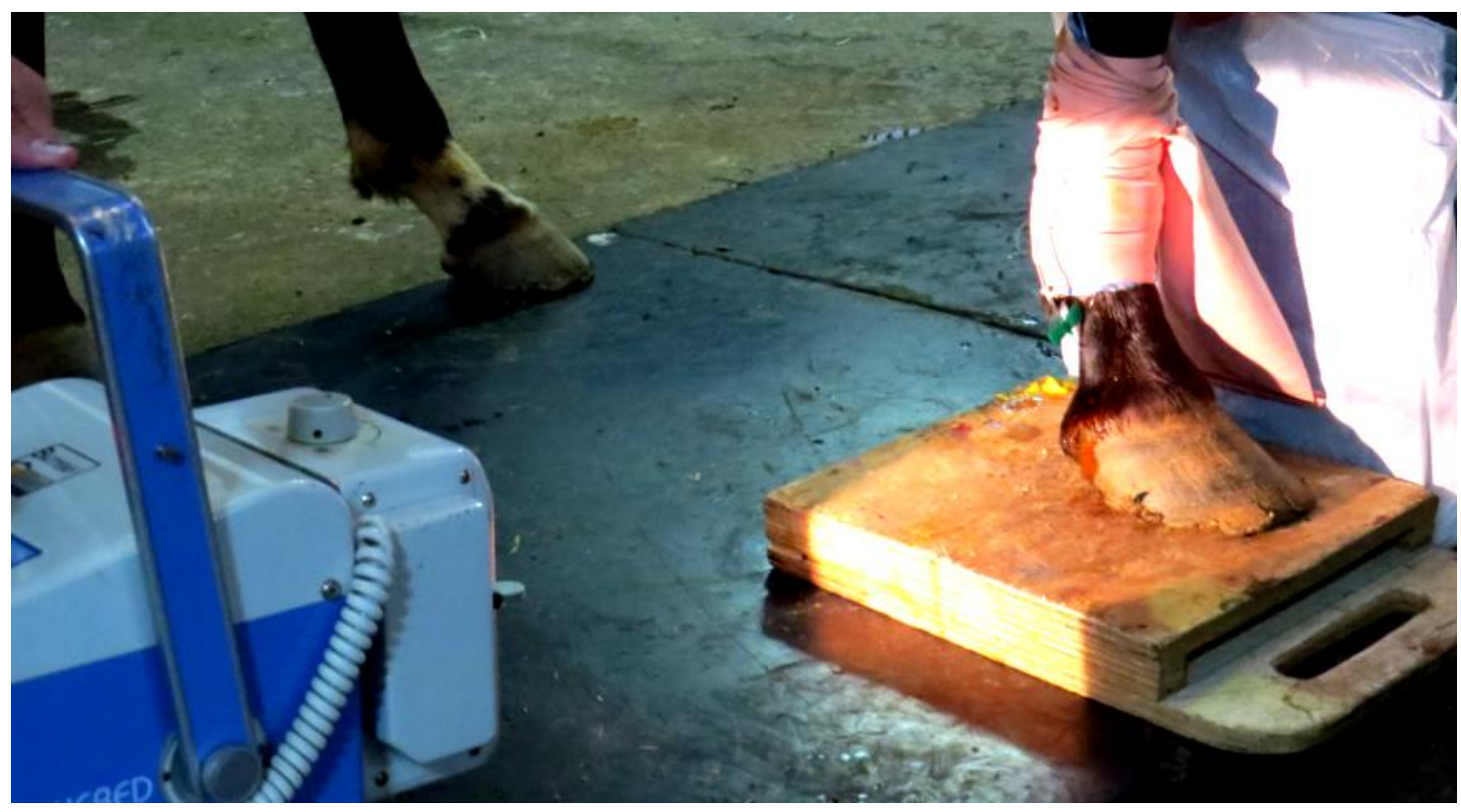

Figura 3: Projeção LM de membro torácico direito de equino após injeção de contraste à base de diatrozoato de meglumina.

Para avaliação das imagens radiográficas obtidas pela venografia, criou-se um sistema de graduação considerando-se o alcance do contraste em cinco regiões: arco terminal, vasos laminares dorsais a falange distal, plexo coronário, vasos circunflexos e bulbo do talão.

Nessa proposta, cada uma das cinco regiões descritas foi quantificada com valores 0 , 1, 2 de acordo com o preenchimento dos vasos: 0 (zero) ausência de preenchimento regional; 1 preenchimento regional parcial $(<50 \%)$ e 2 bom preenchimento regional $(>50 \%)$. Para a referida quantificação do preenchimento das regiões citadas, três examinadores qualificados, foram convidados a avaliar os venogramas, sem que tivessem participado dos procedimentos anteriores. A pontuação máxima obtida por região e por examinador foi de 2 , ou seja, região que apresentou alcance de contraste acima de 50\%. Se cada região obtiver pontuação máxima, a somatória das 5 regiões seria de 10 pontos por examinador. Caso os 3 examinadores atribuíssem 10 pontos na avaliação de determinado dígito, este ficaria com 30 pontos no total. É importante salientar que houve $1 \%$ de diferença de julgamento e de pontuação entre os examinadores.

Os valores obtidos através da análise dos venogramas e atribuição de pontuações para os dígitos torácicos de equinos claudicantes e não claudicantes foram submetidos à análise 
estatística ANOVA, complementados por TUKEY, com nível de significância $\mathrm{p}<0,05$ para comparação entre todos os grupos (grupo 1 - não claudicante: membro torácico esquerdo e membro torácico direito, grupo 2 - claudicante: membro torácico esquerdo e membro torácico direito).

\section{RESULTADOS E DISCUSSÃO}

Em virtude da importância do sistema locomotor, dos membros torácicos e da alta frequência de afecções distais nesta espécie, buscou-se colocar em prática a análise de venogramas de animais claudicantes e não claudicantes.

No exame clinico do sistema locomotor, diagnosticou-se onze animais sem claudicação, grau 0/5 (Grupo 1) e oito animais com claudicação de apoio, em um dos membros torácicos, grau 1 a 4/5 (Grupo 2). Para compor a análise, cinco regiões foram analisadas quanto ao alcance do contraste.

Na Figura 4, em (1) é possível visualizar as regiões utilizadas na análise (A) - arco terminal, (B) - vasos laminares dorsais, (C) - plexo coronário, (D) - vasos circunflexos e (E) - bulbo do talão. Neste caso é possível observar que todas as regiões mencionadas tiveram alcance de contraste maior que $50 \%$ sendo atribuído neste caso, a pontuação 2 por região, totalizando 10 pontos por examinador e 30 pontos quando os 3 examinadores foram considerados. Já em (2) observa-se que a região (B) e a região (C) marcada por setas, já não apresentaram bom preenchimento regional. Neste caso, se considerarmos 1 ponto para cada região, o máximo que este digito recebeu de pontuação foi 8 pontos por examinador e 24 pontos após somatória dos 3 examinadores. Em (3) observa-se que as regiões (C) e (E) coraram parcialmente, ganhando 1 ponto por região e que as regiões (A), (B) e (D) não coraram, não somando nenhum ponto. Neste caso, o máximo que o digito em questão somou foi 2 pontos por examinador e 6 pontos, após somatória dos 3 examinadores. 


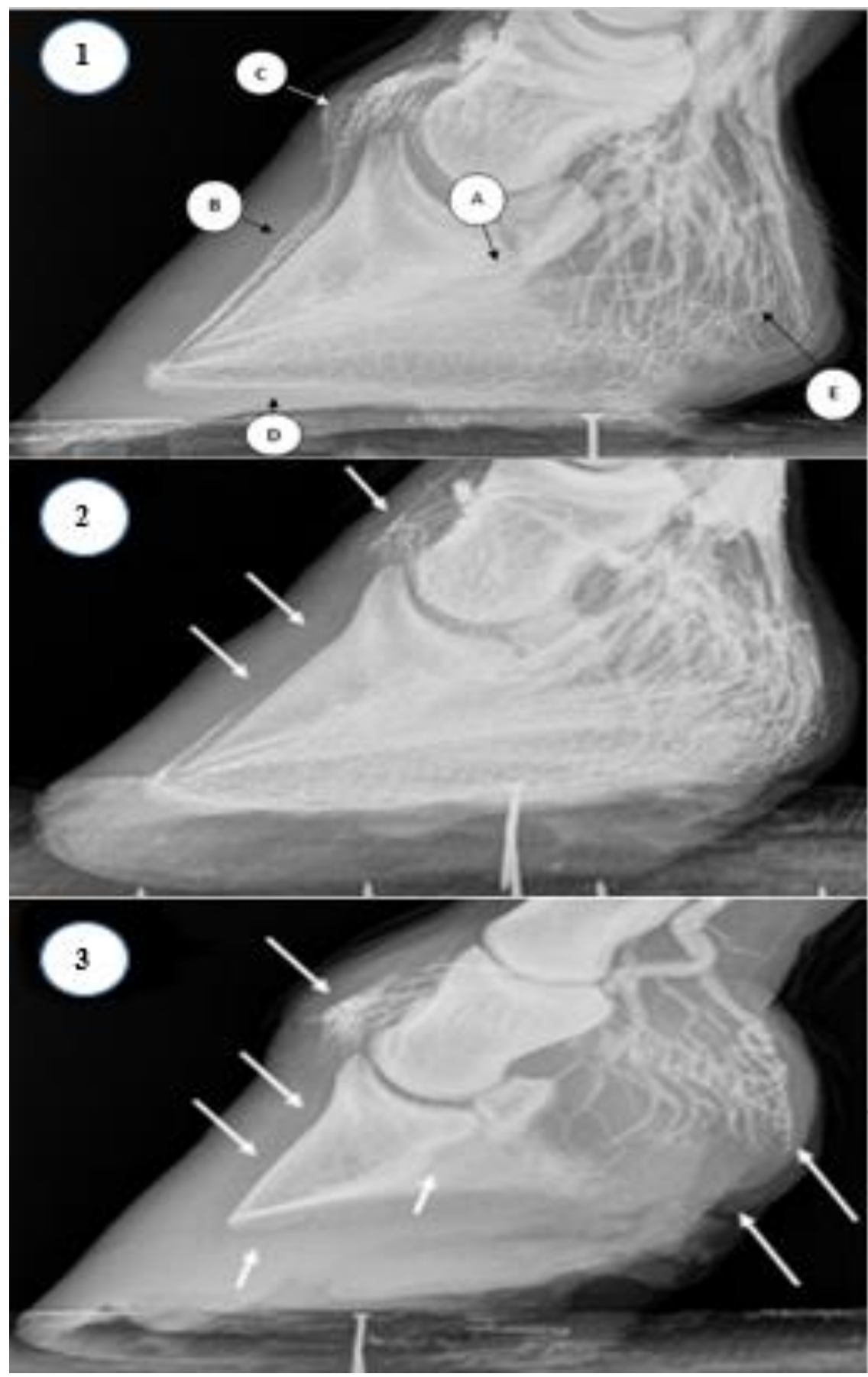

Figura 4: Membros torácicos de equinos adultos não claudicantes (1) e claudicantes $(2,3)$ em projeção lateromedial. (1) Regiões de referência identificadas nos venogramas. (A) - arco terminal, (B) vasos laminares dorsais, (C) plexo coronário, (D) - vasos circunflexos e (E) - bulbo do talão. (2) - Venograma de membro torácico direito com diminuição de preenchimento em plexo coronário e vasos laminares dorsais. (3) Venograma de membro torácico direito com diminuição de preenchimento em plexo coronário, vasos laminares dorsais, vasos circunflexos, arco terminal e bulbo do talão. 
A partir da criação do sistema de pontuação supracitado, que refletiu uma escala semiquantitativa de avaliação de índice de perfusão do dígito por contraste radiográfico, os membros torácicos dos animais do Grupo 1 (não claudicante), foram comparados entre si e com os membros torácicos dos animais do Grupo 2 (claudicante), os quais também foram comparados entre si.

Houve diferença significativa entre os grupos $\left(\mathrm{F}_{3,34}=12,39, \mathrm{p}=0.00001\right)$ quando os animais do Grupo 1 (não claudicantes), tiveram os venogramas dos membros comparados com os do Grupo 2 (claudicantes). É interessante salientar que tanto o membro torácico esquerdo quanto o membro torácico direito apresentaram diferença estatística (Figura 5).

Os membros torácicos dos animais não claudicantes (Grupo 1) não apresentaram diferença estatística entre si (Tukey, $\mathrm{p}=0.9932$ ), visto que neste caso, estes animais eram hígidos e não possuíam distribuição desigual do peso entre os dois membros. O mesmo foi observado na comparação dos venogramas de membros torácicos de animais claudicantes (Grupo 2) (Turkey, $\mathrm{p}=0.3887$ ). Neste caso, por apresentarem distribuição desigual do peso, os animais acabavam sobrecarregando o membro "hígido" o qual passava a apresentar pontuações mais baixas também. Esse fato corrobora Stashak (1994), que cita que alguns equinos com afecções podais podem desenvolver claudicação complementar no membro contralateral, devido ao constante apoio que o animal fará no membro não claudicante visando aliviar o membro claudicante. Observou-se que apenas um animal claudicante do estudo apresentou o membro contralateral totalmente preenchido.

As comparações entre: membro torácico direito de não claudicantes e membro torácico direito de claudicantes (Tukey, $\mathrm{p}=0.00006$ ), membro torácico esquerdo de não claudicantes e membro torácico esquerdo de claudicantes (Turkey, $\mathrm{p}=0.0162$ ), membro torácico esquerdo de não claudicantes e membro torácico direito de claudicantes (Turkey, $\mathrm{p}=0.0001$ ) e entre membro torácico direito de não claudicantes e membro torácico esquerdo de claudicantes (Turkey, $\mathrm{p}=0.0086$ ) apresentaram diferença estatística. 


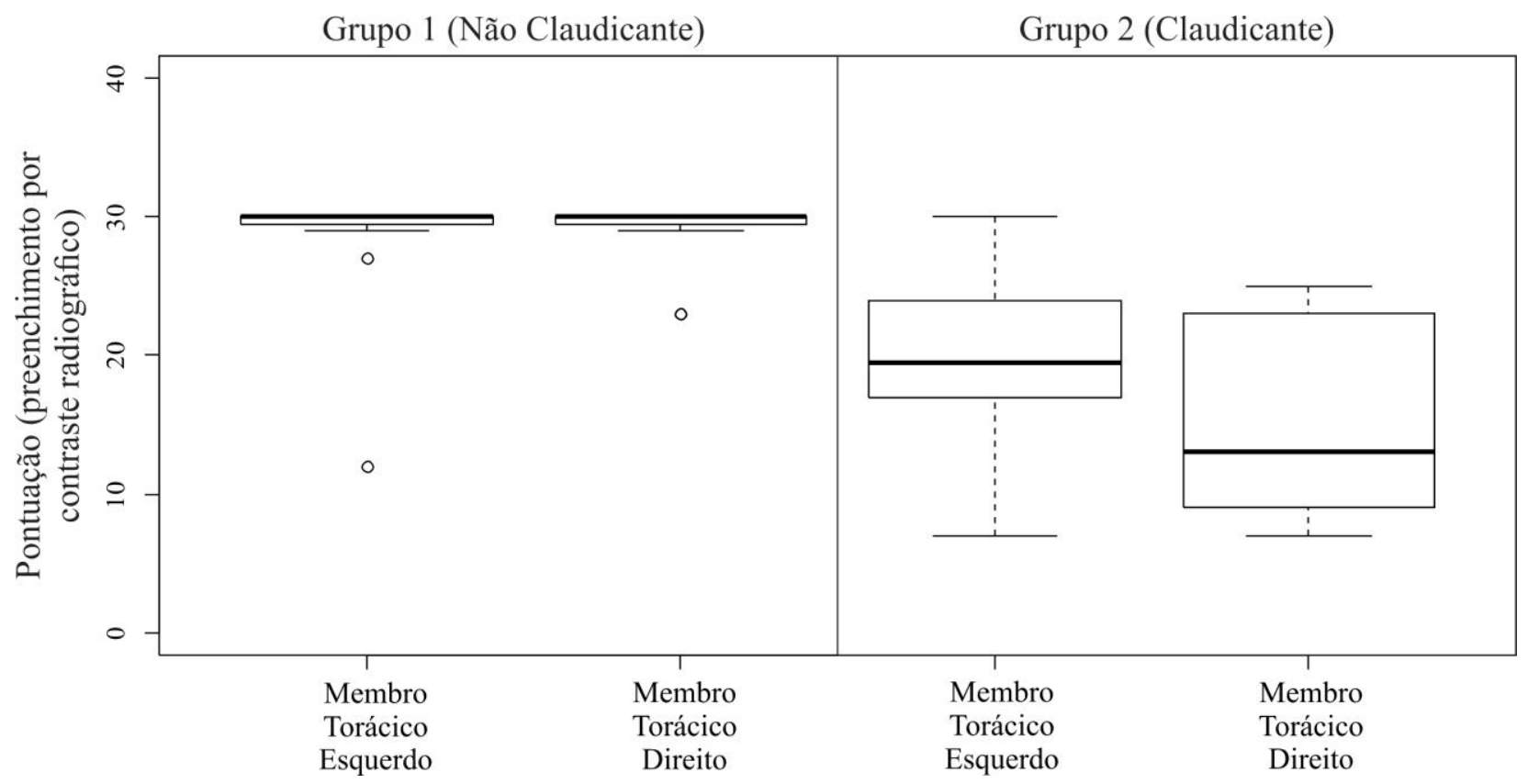

Figura 5: Representações gráficas (diagramas em caixas) das médias e desvios padrões do preenchimento por contraste radiográfico dos dígitos de equinos não claudicantes (Grupo 1) e claudicantes (Grupo 2).

Contudo, foi observado que os animais que mancavam tinham diminuição de irrigação do digito, que se refletiu na escala semi-quantitativa de avaliação de índice de irrigação pelo preenchimento dos vasos circulatórios por contraste radiográfico, como membros que possuíram pontuações menores quando comparados com membros de animais hígidos (não claudicantes). Os valores mais baixos de pontuação alcançados estão diretamente relacionados com a claudicação de apoio.

Nessa situação, devido à falta de contato com o solo, o casco não desempenha algumas de suas funções como, por exemplo, absorção de impacto com o solo, suporte de peso do animal, resistência ao desgaste e auxilio no retorno sanguíneo da extremidade do membro locomotor (KANE et al, 1996; NICOLETTI et al., 2000).

A presença de grande volume de sangue dentro do casco é necessária para que a pressão do fluido amorteça o impacto inicial com o solo. Pode-se argumentar que a estrutura porosa da falange distal tem a função de reter um grande volume de sangue para proporcionar este efeito de absorção de choque (DAVIES et al., 2007).

A expansão lateral do casco e das cartilagens, aumenta a pressão nos plexos venosos, que funcionam como amortecedores hidráulicos forçando o sangue proximal dentro das veias 
digitais, porém, quando o casco está fora do chão, válvulas estratégicas impedem o retorno do sangue ao casco (POLLITT, 1992; PARKS, 2003).

Vale destacar que as pontuações observadas nos animais claudicantes independeram do grau de claudicação observado, ou seja, não necessariamente, um animal com grau de claudicação 4/5 obteve índices de irrigação podal menores que um animal com grau 3/5 (Figura 6).

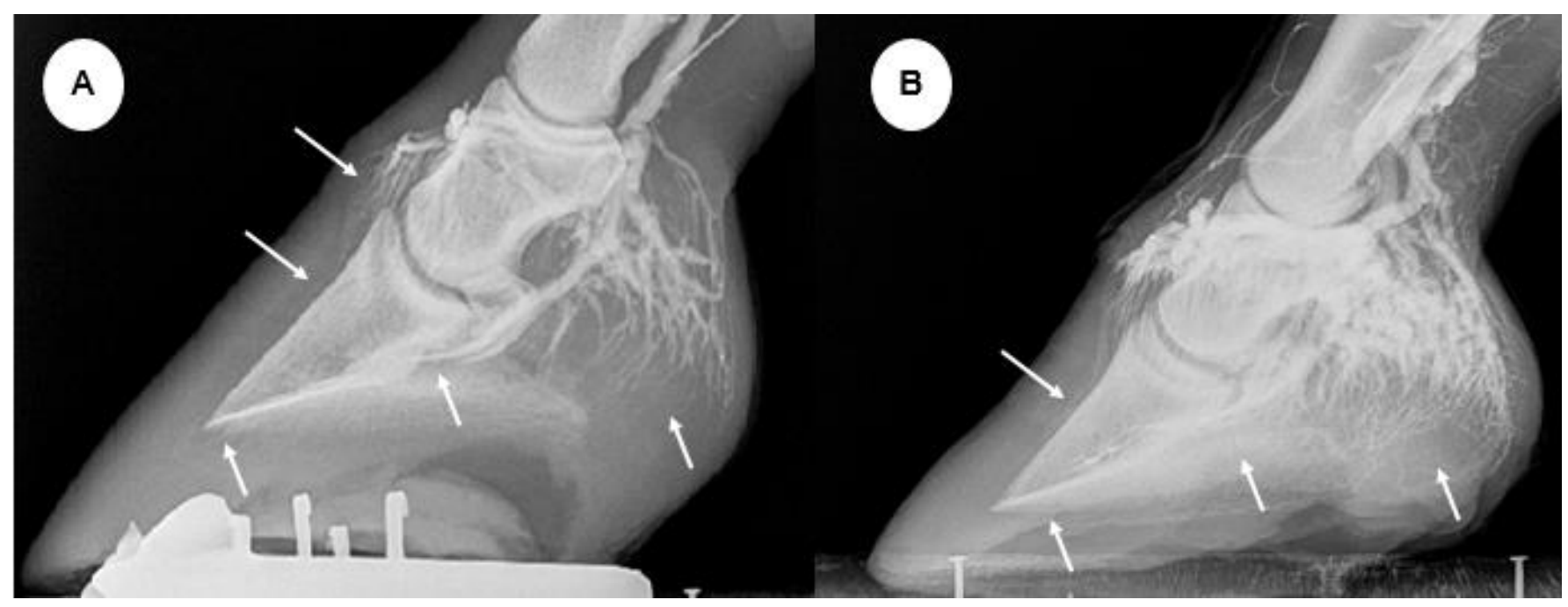

Figura 6: Venograma do membro torácico direito de animal com claudicação 3/5 com pontuação total de 8 pontos (A), venograma do membro torácico esquerdo de animal com claudicação 4/5 com pontuação total de 16 pontos (B). Setas apontam diminuição de perfusão, em (A): plexo coronário, vasos laminares dorsais, vasos circunflexos, arco terminal e bulbo do talão; em (B): vasos laminares dorsais, vasos circunflexos, arco terminal e bulbo do talão.

Quanto as regiões observadas, a diminuição de irrigação se fez presente no arco terminal (8/8), nos vasos circunflexos (8/8), nos vasos laminares dorsais $(7 / 8)$, plexo coronário $(5 / 8)$ e bulbo do talão $(2 / 8)$.

Todos os animais do Grupo 2 tiveram algum grau de diminuição de perfusão na região do arco terminal. A constatação deste fato chama a atenção e alerta a seriedade deste fato, pois segundo D’Arpe \& Bernardini (2008), a integridade desta região é crucial para manter o dígito vivo. Na laminite crônica, o arco terminal, torna-se eventualmente vulnerável e a isquemia pode ocorrer (RUCKER, 2007). Tal fato corrobora o presente trabalho, visto que metade dos animais possuíam laminite crônica. Quanto aos outros quatro animais; um apresentava pododermatite séptica e posteriormente foi submetido a cirurgia na qual foi 
constatada necrose da cartilagem alar e regiões adjacentes; outro apresentava síndrome do navicular e os outros dois não tiveram o diagnóstico fechado.

Todos os animais do estudo em questão apresentaram déficit de preenchimento na região dos vasos circunflexos, que segundo Rucker (2007) é na maioria das vezes a primeira do digito a ficar comprometida no início da laminite.

A maioria dos animais (87\%) possuíam falha de perfusão na região dos vasos laminares dorsais. Tal fato pode ser explicado pela relação direta deles para com o arco terminal (RUCKER, 2007). Assim, os vasos laminares dorsais são perfundidos por ramos provenientes do arco terminal. Pôde-se constatar bem o fato da direção do fluxo no venograma do animal com necrose de cartilagem alar, ou seja, não houve preenchimento no arco terminal e consequentemente não houve também nos vasos laminares dorsais corroborando.

O plexo coronário não foi preenchido em mais da metade dos animais (63\%), revelando que uma de suas funções principais, válvula de segurança para a pressão hidrostática e dinâmica (D’ARPE \& BERNARDINI, 2008), quando pensa-se na drenagem do dígito não estava sendo realizada adequadamente

A região do bulbo do talão apresentou déficit de perfusão em $25 \%$ dos animais claudicantes do estudo, este dado corrobora Rucker (2007) e D’Arpe \& Bernardini (2008), que citaram que a perfusão sanguínea nos talões é raramente reduzida. Isso se deve ao percurso da artéria bulbar (palmar ao metacarpo) e ao fato de que a maioria do dano tecidual e carga mecânica do digito ocorre na região anterior da parede do casco.

Arthur \& Rucker (2003) citaram que o plexo coronário, os vasos laminares dorsais e os vasos circunflexos são áreas que estão consistentemente normais em cavalos que não apresentam laminite, porém anormais em animais portadores de laminite crônica. Essa informação corroborou parcialmente com o presente estudo. Os animais com claudicação de apoio de forma geral não tiveram vasos laminares dorsais e vasos circunflexos perfundidos, não somente os com quadro clínico de laminite. Além disso, nos casos observados de laminite crônica, não foram todos os animais que apresentaram baixa perfusão na região do plexo coronário, sendo a região do arco terminal mais acometida em relação a outra (Figura 7). 


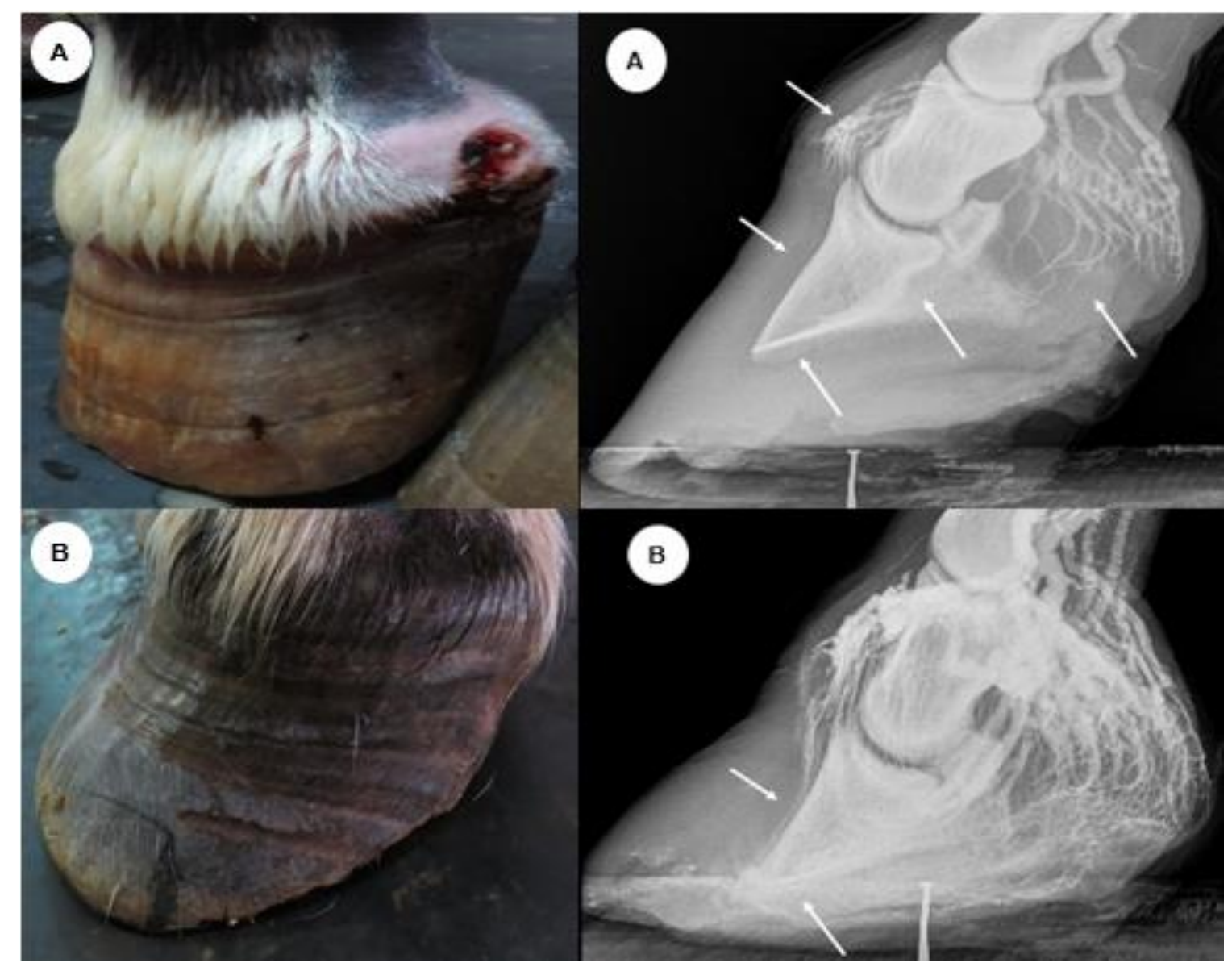

Figura 7: Fotos e venogramas dos membros torácicos direitos de dois animais claudicantes. (A) animal com necrose de cartilagem alar. Setas apontam diminuição de perfusão em plexo coronário, vasos laminares dorsais, vasos circunflexos, arco terminal e bulbo do talão. (B) animal com laminite crônica. Setas apontam diminuição de perfusão em vasos laminares dorsais e vasos circunflexos.

Em relação a angulação do casco, todos os animais, claudicantes e não claudicantes foram classificados, de acordo com a angulação obtida na pinça do casco em: encastelados $\left(>54^{\circ}\right)$, achinelados $\left(35-49^{\circ}\right)$ e normais $\left(<54^{\circ}\right)$ (Figura 8). A angulação para cascos normais inferior a 54 condiz com Bushe et al. (1987) e Stashak (1994) que citaram valores de 45-50 . 


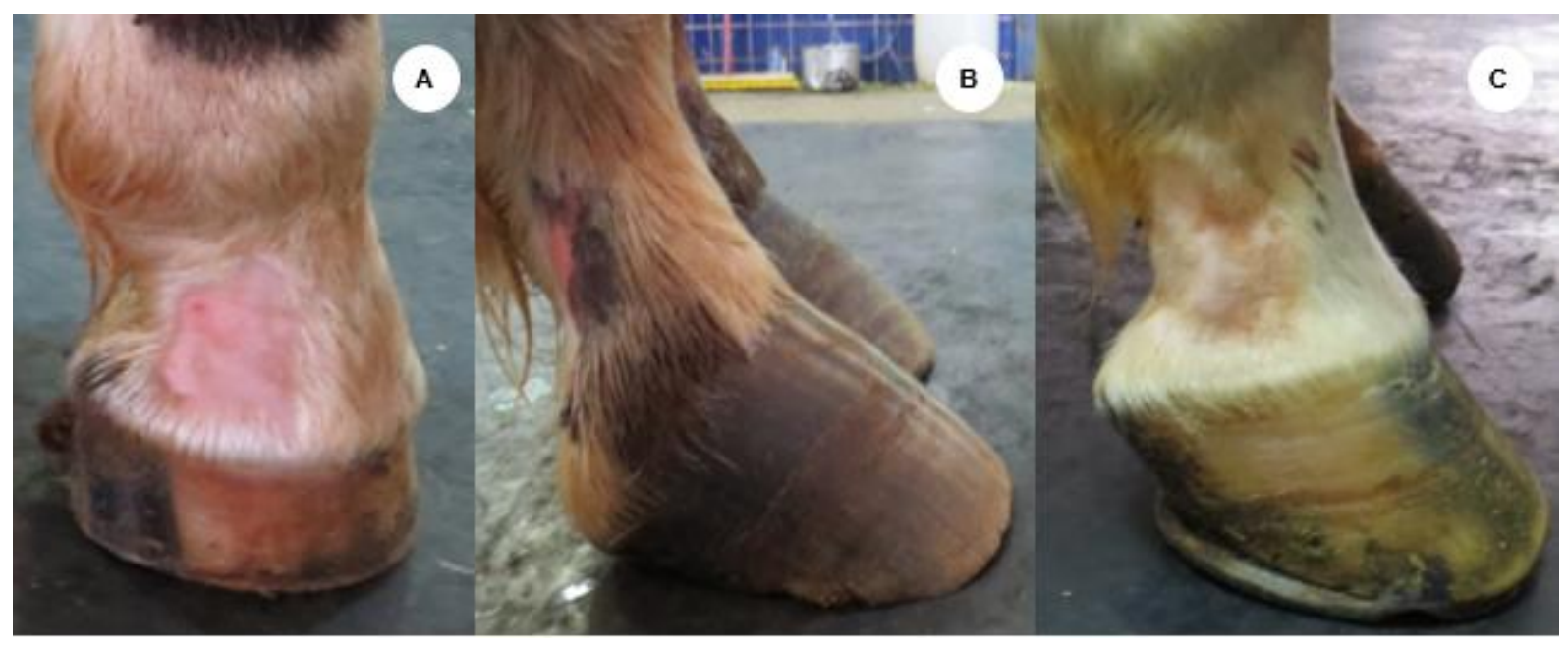

Figura 8: Conformação do casco de três animais. (A) casco encastelado, (B) casco achinelado, (C) casco normal.

Observou-se que os animais claudicantes que tinham angulação do casco normal ou achinelada tendiam a pontuações maiores em relação aos animais encastelados; corroborando Frandson et al (1978), Clayton (1987) e Barey (1990) que relataram que o ângulo também influencia a concussão no dígito, sendo que quanto maior o ângulo menor a concussão. Em decorrência desse apoio desproporcional do peso, ocorre também um efeito de separação entre os talões que, por sua vez, causa um colapso mecânico das estruturas que sustentam o bulbo do talão (TURNER \& STORK, 1988; STASHAK, 1994).

Nos integrantes do Grupo 1 (não claudicantes), o fato do animal possuir casco de conformação achinelada, normal ou encastelada não contribuiu para uma diminuição de perfusão no venograma. Possivelmente neste caso, além destes animais já serem adaptados à conformação do casco não existia o efeito somatório da claudicação de apoio que fazia com que os cascos não desempenhassem suas funções adequadamente, devido à falta de apoio constante do casco com o solo. 


\section{CONCLUSÃO}

Com base nos achados do presente trabalho concluiu-se que a deficiência de apoio reduziu a perfusão do digito, sendo que o grau de claudicação não teve relação com a diminuição de preenchimento no exame radiográfico. Nos venogramas de animais claudicantes, a diminuição de irrigação se fez presente com maior frequência no arco terminal, nos vasos circunflexos e nos vasos laminares dorsais.

Animais claudicantes com angulação do casco normal ou achinelada apresentaram pontuações maiores em relação aos animais encastelados. Esse fato já não ocorreu com os animais não claudicantes no qual angulações diferentes não influenciaram na perfusão digital. 


\section{REFERÊNCIAS BIBLIOGRÁFICAS}

AAEP. Guide for veterinary service and judging of equestrian events. 5ed. Lexington, KY: American Association of Equine Practitioners, 63p. 1996.

ARTHUR, E.G., RUCKER, A. The use of digital venography for assessment of perfusion déficits in laminitis. Poster Presentation, University of Missouri, MU chapter of Phi Zeta, 2003.

BAREY, E. Investigation of the vertical hoof force distribution in the equine forelimb with an instrumented bone-foot. Equine Veterinary Journal, s. 9, p. 35-38, 1990.

BAXTER, G. M. Adams and Stashak's Lameness in Horses. Wiley, 2011.

BUShE, T.; TURNER, T. A.; PAULOS, P. W.; HARWELL, N. M. The effect of hoof angle oon coffin, pastern and fetlock joint angles. In: Annual Convention of the American Association Equine Practitioners, 33, New Orleans. Proceedings... New Orleans: AAEP, p. 729-737, 1987.

BURG, E., DIEPENBROEK, C., HOORNEMAN, N.; LICHTENBERG, E. Explorative Study of the Natural Balance Method of Hoof Care in Horses. Wageningen University and Research Center, Wageningen, 2007.

CLAYTON, H. M. Comparision of the stride of trotting horses trimmed with a normal and a broken back hoof axis. In: Annual Convention of the American Association Equine Practitioners, 33, New Orleans. Proceedings... New Orleans: AAEP, p. 289- 298, 1987.

COFFMAN, J.R.; JOHNSON, J. H.; GUFFY, M. M.; FINOCCHIO, E. J. Hoof circulation in equine laminitis. Journal of the American Veterinary Medical Association, v.156, n.1, p.7683, 1970. 
DAVIES, H. M. S.; PLILIP, C. J.; MERRITT, J. S. Functional anatomy of the equine digit: Determining function from structure in Floyd AE, Mansmann RA Equine Podiatry, $1^{\mathrm{a}}$ ed. Saunders, p. 25-41, 2007.

D'ARPE, L; BERNARDINI, D. Interpreting contrast venography in horses with controlateral laminitis. Procedings of the European Society of Veterinary Orthopedics and Traumatology. p. 34-39, 2008.

FRANDSON, R. D.; LABEL, J. L.; SLADE, L. M.; BARBRANA, R. C. Effect of slope of the equine hoof on concussion and phalangial angulation. American Farrier's, v. 4, p. 7273, 1978.

KANE, A. J.; STOVER, S. M.; GARDNER, T. A.; CASE, J.T.; JOHNSON, B.J.; READ, D,H,; ARDANS, A.A. Horseshoe characteristics as possible risk factors for fatal musculoskeletal injury of thoroughbred racehorses. American Journal Veterinary Research, Schaumburg, v. 57, n. 8, p. 1147-1152, 1996.

NICOLETTI, J. L. M.; SCHLEGEL, C.; THOMASSIAN, A. Mensuração do casco de eqüinos para identificação objetiva de anormalidades de conformação. Veterinária Notícias, Uberlândia, v. 6, n.1, p. 61-68, 2000.

PARKS, A. Form and function of the equine digit. The Veterinary Clinics: Equine Practice. Elsevier, v.19, p. 285-307, 2003.

POLLITT, C.C. "Laminitis in perspective" in Equine Laminitis current concepts, 1 a ed, RIRDC, 2008.

POLLITT, C. C. Clinical anatomy and physiology of the normal equine foot. Equine Veterinary Education, London, v. 4, n. 5, p. 219-224, 1992.

REDDEN, R.F. A technique for performing digital venography in the standing horse. Equine Veterinary Education, v.13, n.3, p.128-34, 2001. 
ROBINSON, N.E. Digital blood flow, arteriovenous anastomoses and laminitis. Equine Veterinary journal, 22, p. 381-383, 1990.

RUCKER, A. The digital Venogram in Floyd AE, Mansmann RA Equine Podiatry, $1^{\text {a }}$ Edição, Saunders, p. 328-358, 2007.

RUCKER, A. Clinical Applications of Digital Venography. Journal of Equine Veterinary Science, v.30, n.9, p.491-503, 2010.

SPEIRS, V. C. Exame clínico de equinos. Porto Alegre: Editora Artes Médicas Sul Ltda, 366p., 1999.

STASHAK, T.R. Claudicação em equinos segundo Adams, 4ed. São Paulo: Roca, 923p. 1994.

STASHAK, T.R. Claudicação em equinos segundo Adams, 5ed. São Paulo: Roca, p. 1015$1071,2006$.

STOKES, A.M.; EADES, S.C.; MOORE, R.M. Pathophysiology and treatment of acute laminitis. In S.M. Reed, W.M. Bayly \& D.C. Sellon, Equine internal medicine, 2ed. Saunders: St. Louis, USA. p. 522 - 530, 2004.

THOMASSIAN, A. Fisiopatologia e tratamento da laminite em equinos. Revista de educação Continuada do CRMV-SP, v.3, n.2, p.16-29, 2000.

TURNER, T.A.; STORK, C. Hoof abnormalities and their relation to lameness. In: American Association of Equine Practitioners,32. Proceedings..., v. 34, p. 293-297, 1988. 